Volume 8, No.1.6, 2019

International Journal of Advanced Trends in Computer Science and Engineering

Available Online at http://www.warse.org/IJATCSE/static/pdf/file/ijatcse7181.62019.pdf

https://doi.org/10.30534/ijatcse/2019/7181.62019

\title{
Mapping from wheeled locomotion to vertical group of joint space for snake like robot rectilinear locomotion
}

\author{
Samsi Md Said ${ }^{1}$, Amir Sharizam Ismail ${ }^{2}$, Tengku Mohd Azahar Tuan Dir ${ }^{3}$, Jamel Othman ${ }^{4}$, Norazlin \\ Ibrahim $^{5}$ \\ Industrial Automation Section, Universiti Kuala Lumpur Malaysia France Institute, 43650 Bandar Baru Bangi \\ Selangor, Malaysia \\ samsi@unikl.edu.my, amirsharizam@unikl.edu.my, tgazahar@unikl.edu.my, jamel@unikl.edu.my, \\ norazlin@unikl.edu.my
}

\begin{abstract}
This paper presents mapping from friction roller to robot joint space for complex snake like robot gait such as rectilinear locomotion. In this study, the parametric periodic wave is generated quantitatively from friction roller model to implement locomotion for hyper redundant robot multiple joint and linkages or joint space world. This procedure is called the mapping from operational or world to robot joint space. The body shape can be modelled as fraction of ideal diameter of the whole length of snake like robot body enveloping a desired diameter. The body shape, speed and contact points can be determined from the approximation of one whole diameter and desired diameter planned by the motion planner.
\end{abstract}

Key words: Mapping from world to joint space; Rectilinear Gait; Orthogonal Configuration Robot.

\section{INTRODUCTION}

Robotic link and joint can be treated at system level and joint level. System level can be considered high level while at joint space can be considered low level. Low level robot arm focus on controller design. Eduardo I. et all [1] design four DOF pseudo-anthropomorphic robotic arm having shoulder yaw and pitch, elbow pitch and wrist pitch. The motion capture device used is the Microsoft Kinect which detects its operator's arm position. S.V.R.K.Rao et. all [2] develop Brain computer Interface to control portable robot arm.At joint level, A.A.M Zahir et all. [3] used GA to optimize PI controller to regulate speed of cart follower.Robotic arm system can be divided further to mobile and static system. Snake robot can be considered as mobile robotic system. Snake like robot capability to enter narrow space is very useful since narrow space exist either man made (inside aircraft wing or engine), in nature (endoscopy operation for low opening surgery) and disaster areas.

\section{LITERATURE REVIEW}

Even though the rectilinear gait is more less space consuming in horizontal and vertical direction, most of literature focused type of motion which took large floor area due to serpentine and sidewinding motion most dominant type of locomotion seen in natural snake [3]-[10]. The snake like robot capability to enter narrow space is very useful since narrow space exist either man made (inside aircraft wing or engine), in nature (endoscopy operation for low opening surgery) and disaster areas [11]-[14]. It is estimated that the role of snake like robot as important as today industrial robot for service industry which will capture 7 billion USD worth of market in 2021 [14].

Md. Raisuddin Khan et. al proposed wriggler gait and gait transition from serpentine to wriggler for entering narrow space by considering snake scale but in lateral mode by employing inverse kinematic [20]-[21]. Norzalilah Mohamad Nor and Shugen $\mathrm{Ma}$ introduce linear bipolar activation function in Central Pattern Generator (CPG) to control the smooth transition the number of $\mathrm{S}$ body shape for serpentine locomotion for entering and adapting to various narrow space width and to avoid external obstacle [22]-[23]. Both works improve the top view serpentine gait by employing Inverse kinematic and CPG for multi configuration snake like robot adapting to narrow space. Several researchers focused on side view locomotion such as rectilinear snake like robot to derive the ability of snake moving into confined space [15]-[19]. The problem of rectilinear gait snake like robot is different mechanical design snake like robots are proposed. There is no uniform design universally used for rectilinear snake like robot. Second, special design using prismatic or sliding joint is employed for rectilinear gait. Prismatic joint is more complex, expensive to build and to maintain in lieu of revolute joint which possess more dexterity and compactness for robotic design method [24]. Third, the sliding prismatic joint creates friction with floor space which causes the need for special anchor points to balance horizontal friction force [25]. In the real field, the coefficient of friction may be very large, and the movement will be stuck which raise the requirement for movement that free from friction or obstacle avoidance movement. Fourth, anchor or pivot points exist that act like stopper to propel rectilinearly the segment of snake robot from tail, middle segment and head. The pivot or anchor point become a major study such as in rectilinear snake like locomotion robot especially in exaggerated linear gait snake 
like robot that employ variable friction anchor point developed by Hopkins [26]. Three segment rectilinear gaits considering rough surface with dry friction which is scalable into higher degree of freedom robot is introduced by Wenbin Tang and Shugen Ma in [27].

The advantages of employing sliding joints are due to reducing the height signature of snake robot. By using revolute joint the side view profile may be larger and defeat the purpose of employing snake like robot moving into confined space. It is necessary to trade of between unavoidable height profile and friction free forward movement by proposing multi-configuration design using revolute joint that adapt into contour side view profile. To solve problem of too many rectilinear gaits dedicated snake like robot, an orthogonal configuration snake like robot is being proposed in this work. Orthogonal configuration Snake like robot is among the important design since it can perform one plane vertical (rectilinear gait) or horizontal (serpentine) and three-dimensional plane (sidewinding and rolling) [28]-[30]. These measures solve the rectilinear gait friction problem but maintain versatility of orthogonal snake like robot configuration for turning and three-dimension locomotion if needed to bridge to the rectilinear movements. Motoyasu Tanaka and Kazuo Tanaka [31] use orthogonal configuration snake like robot with wheel using pitch axes to control ascending and descending step and using yaw axes for directional control for multi-step rectilinear gait locomotion. Sine wave is being used to generate rectilinear vertical plane locomotion in orthogonal design snake like robot by controlling the amplitude or joint angle and phase angle between adjacent pitch joint [32]. Using sine wave is under approach of traveling waves which creates impact to the ground and body lifting from flat world which cause high torque and energy lost which is unneeded for forward motion.

Parameter determination in joint space snake robot rectilinear and concertina gait planning is very important since amplitude decide the maximum and minimum cyclic angular joint distance, frequency decide the overall speed and phase angle decide the overall movement (otherwise snake like robot will stay in one place without nett displacement). However, current cyclic planning still uses intuitive approximation for the abovementioned parametric determination. Thus, the friction roller mapping will be proposed here where the exact sine wave parametric property such as amplitude, frequency, and phase angle at every joint numbered from first joint to final joint can be determined from wheeled locomotion (friction roller) world. This exact conversion procedure will help designer from depending on intuitive and iterative effort in order to plan a rectilinear and concertina gait for hyper redundant robot system. So, this chapter will discuss about the algorithm from wheeled locomotion (friction roller) to linkages world (joint space).

\section{METHODOLOGY}

Friction roller of certain radius or diameter when being rotated at certain angle and speed can produce certain translational distance and speed as shown in equation 1 and 2 . One complete rotation of friction roller rotation at time $T$, produce distance and speed as in equation 3 and 4 . So in order to find speed we need to decide the appropriate radius $R$ and angular speed $\omega$ to produce one rotation cycle time $T$. The determination of speed can solve the translational distance per unit time issue in implementing basic land vehicle journey planning. Thus, equation 1 to 5 is the main equation govern the desired distance and speed in friction roller world which can easily planned by designer since friction roller is quite common as in wheeled robot or land transport vehicle. The total distance desired $d_{t}$, at certain desired time $T_{t}$, as in equation 5, are the parameter of the planner or planner input or design parameter in friction roller world. Planner also need to approximate the value of friction roller diameter $D_{T}$ since the height or swaying up and down for rectilinear or concertina gait in joint space which consume a lot of power to overcome gravity is governed by value of $D_{t}$.

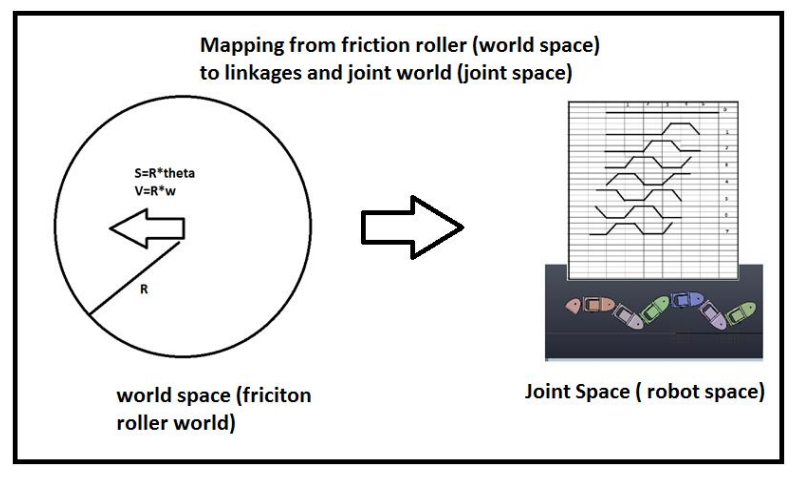

Figure 1: Friction roller to linkage kinematic mapping.

$$
\begin{aligned}
& x(t)=\alpha R \\
& v=\frac{d x}{d t}=\frac{d x}{d t} R \\
& d_{c}=2 \pi R \\
& v_{v}=\frac{2 \pi R}{T}=\omega R \\
& v_{a v}=\frac{D_{t}}{T}
\end{aligned}
$$

In order to decide the number of links enveloping friction roller radius $R$ or diameter $D$, enveloping links technique is proposed here. A multi-joint of links enveloping a circle method is proposed in figure 2. For example, the rectangular enclosure enveloping a friction roller circle, the value of $\boldsymbol{R}$ is seen as half of robotic link $L$ or equal to $D$ since $R$ is equal 
to half of $\boldsymbol{D}$. To model for hexagon and other number of links enveloping a circle of friction roller, a general Link to radius relationship can be represented in equation 6 and 7 where $n$ the number of links (or degree of freedom ) enveloping circle of radius $R$ or diameter $D$ and $L$ is the value of link length. If the number of link enclosing a circle is $n=4$ as in rectangle enclosing a circle, then the value of $\boldsymbol{R}$ or $D$ can be approximated from equation 6 and 7 . With the determination of friction roller $R$ (from known value of link length $L$ and previous planner desired average speed from equation 5 , the friction roller one revolution cycle time $T$ and angular speed $\omega$ can be decided in equation 10 and 11. Maximum and minimum joint angle for every linkage joint also related to enveloping links technique in figure 2. Equation 12 describe the maximum joint angle in positive and negative term which is one rotation degree divided by number of links enveloping a circle. So the design parameter can be reduced into simplified time taken $T$ for one friction roller one cycle rotation (in second) and its related angular speed ( $\mathrm{rad} / \mathrm{sec})$ and maximum joint angle (positive and negative) $\theta_{\max }$ as in equation 10,11 and 12. Phase angle between multi joint system of hyper redundant robot also similar to maximum and minimum joint angle and represented in equation 13. It is clear from equation 12 and 13 that approximation of diameter $D$ and value of link $L$, will decide the value of maximum angle $\theta_{\max }$ and phase angle $\phi_{\text {phase }}$.

The input output relationship to summarize this approach is displayed in figure 3. Designer need to approximate the value of design parameter for rectilinear gait such as operational diameter $D_{o p}$, input value of link length $L$ from existing robot link length and the average speed $v_{a v}$. From the design parameter input, the friction roller mapping algorithm will produced the multiple output of performance parameter such as number of links enveloping circle, cycle time $T$, angular speed $\omega$, phase angle $\phi_{\text {phase }}$ and maximum joint angle $\theta_{\max }$. This straightforward conversion is a major contribution of this research which frees the designer from intuitive approximation work of planning the rectilinear and concertina gait. The parametric periodic wave is generated quantitatively from friction roller model to implement locomotion for hyper redundant robot multiple joint and linkages or joint space world. This procedure is called the mapping from operational or world to robot joint space.

The intended operational radius of $D_{o p}$ is not the same with total value of $D_{\text {tot }}$ where the whole body length of hyper redundant (total DOF) robot enveloping maximum diameter.

$$
n L=2 \pi R=\pi D_{o p}
$$

$$
\begin{aligned}
& n=\frac{\pi D_{\alpha p}}{L} \\
& v_{a v}=\frac{R}{2} \omega=\frac{D_{\alpha p}}{4} \omega \\
& \omega=\frac{4 v_{a v}}{D_{a p}} \\
& T=\frac{2 \pi}{\omega} \\
& \omega=\frac{2 \pi}{T} \\
& \theta_{\text {max }}= \pm \frac{2 \pi}{n}= \pm \frac{2 L}{D_{o p}} \\
& \phi_{\text {phase }}=\frac{2 \pi}{n}=\frac{2 L}{D_{o p}}
\end{aligned}
$$

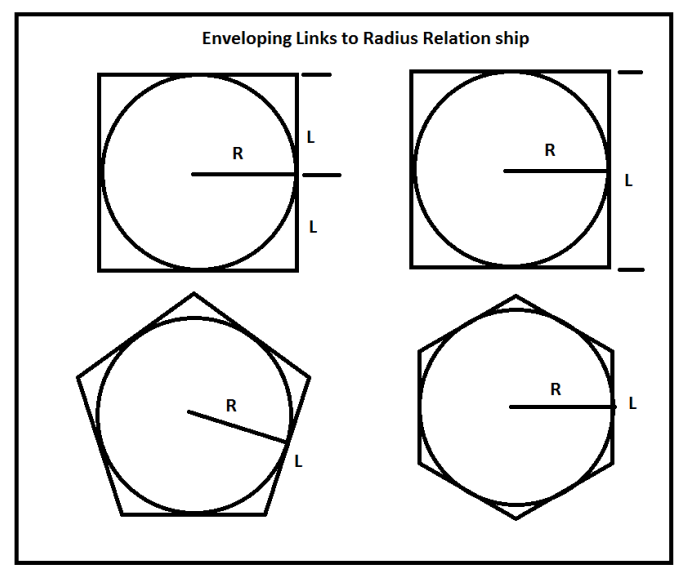

Figure 2: Enveloping links technique to find friction roller $R$ from link value $L$.

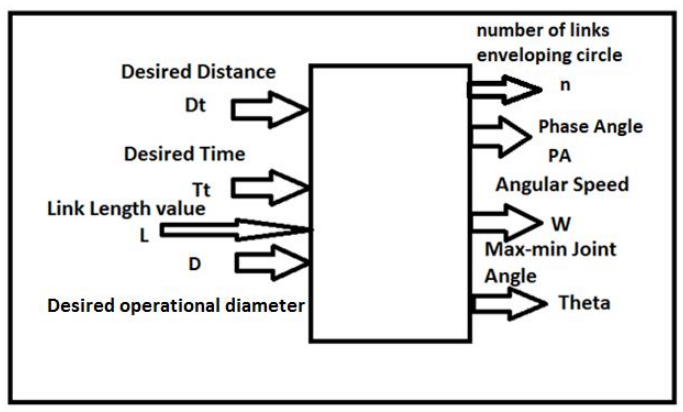

Figure 3: Friction roller input-output relationship

\section{Configuration design and decision}

Here vertical or column pair and horizontal or row pair is introduced. By referring figure 4 , the first joint can be positive if it reside in first row or quadrant III and IV, and possessing the value of negative if it reside in second row or quadrant I and II. If the first column is positive pair, the second column become negative pair and alternately repeated into alternate positive and negative pair joints of positive and negative. This concept elaborated further in figure 5 where joint 1 is in positive region because reside in quadrant III or lower row. Joint 2 and 3 become negative because second column is 
negative value. The joint 4 and 5 is positive value because it belongs in the positive pair. In the second cycle joint 1 also reside in bottom row so joint 1 and 2 have positive value and joint 3 and 4 got negative value in second column and joint 5 and 6 again become positive. In third cycle joint 1 posses negative value since it reside on top row and joint 2 and 3 become negative since it belong to second alternate pair. Joint 4 and 5 again become positive since it alternately switch to positive value. In cycle 4 , joint 1 becomes negative since it resides on top row. If the first pair negative then joint 2 also negative value while joint 3 and four are positive because it belong to alternate second column pair. Joint 5 and 6 again become positive due to this alternation procedure between columns. So the configuration pattern has been proposed in this work through cycle pattern started with the location of joint1. By identifying joint one (1) location, we can determine the joint one sign and subsequent pair sign changes in the next column. Sometime joint one or last joint pair consist only one joint member in certain cycle. What we mean by positive and negative joint is the maximum joint in equation 12 derived previously and shown in figure 6. To accommodate this configuration, simplified and dedicated coordinate assignment system will be proposed in the subsequent paragraph.

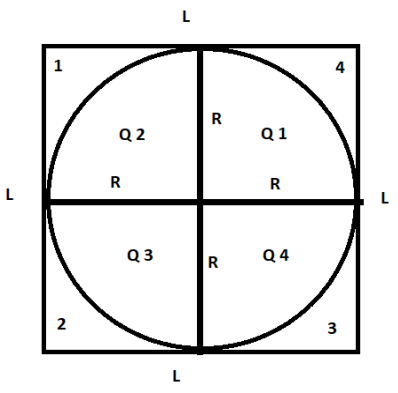

Figure 4: Tail movement from TA to TB

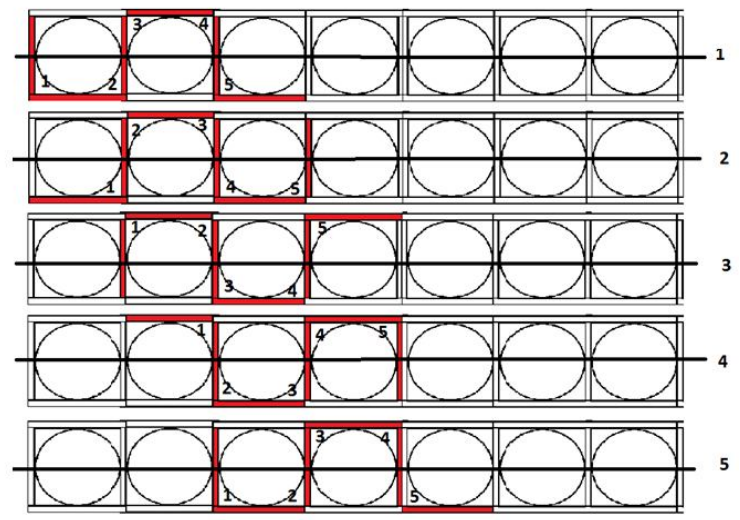

Figure 5: Linkage enclosure to friction roller configuration

\section{Positive and Negative Joint periodic Angle}

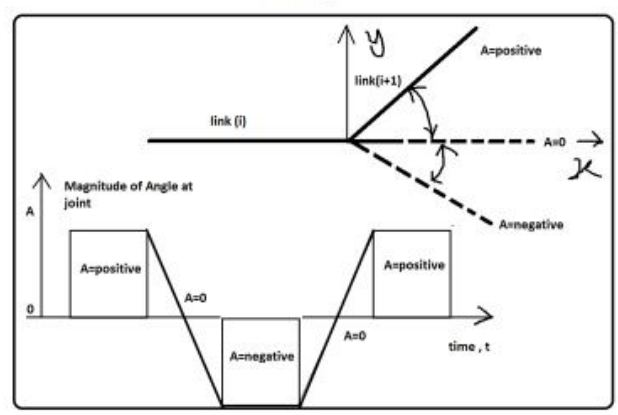

Figure 6: Joint positive and negative nomenclature

\section{Results AND Discussion}

Total length of snake like robot enclosing a virtual circle will produce diameter of certain value $D$ which is the ideal diameter that govern the rectilinear gait locomotion in joint space world. Figure 7 shows a snake like robot with length $L_{\text {tot }}$ equal $1.942 \mathrm{~m}$ and through relation in equation 14 will produce enveloping circle with diameter $D=0.6181$ as in equation 15 . This diameter $D$ in equation 15 will become reference diameter for this mapping from wheeled to joint space locomotion procedure. With some modification, mapping from full circle shown in figure 15 with this diameter will produce one diameter movement as shown theoretically in figure 16. Equation 16 produces every joint angle progressive value of snake like robot toward performing one diameter closed circle configuration that mentioned previously which is $33.6^{\prime \prime}$ degree (as shown in figure 8 and $9)$.
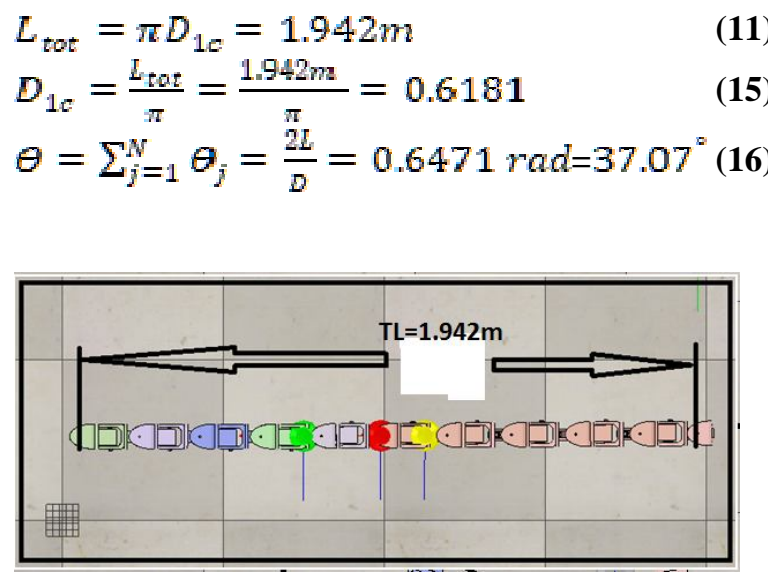

Figure 7: Shows a snake like robot with length $1.942 \mathrm{~m}$ 


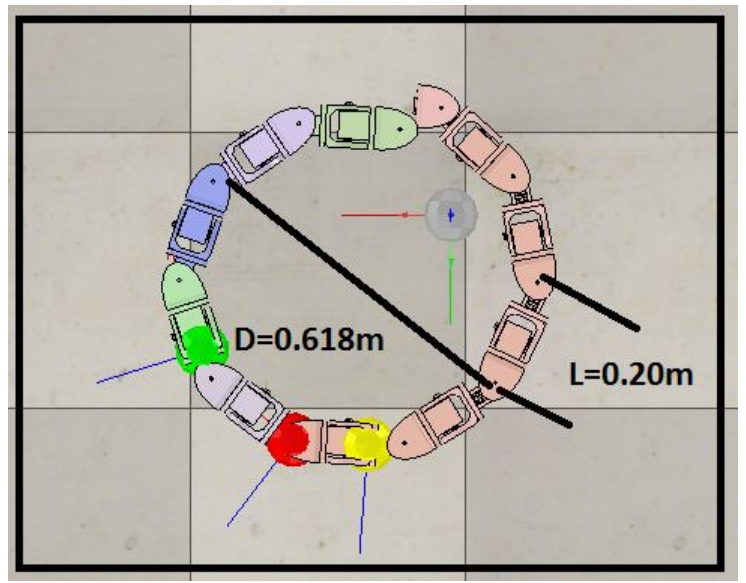

Figure 8: Total length $1.942 \mathrm{~m}$ enveloping circle with diameter $D=0.6181$

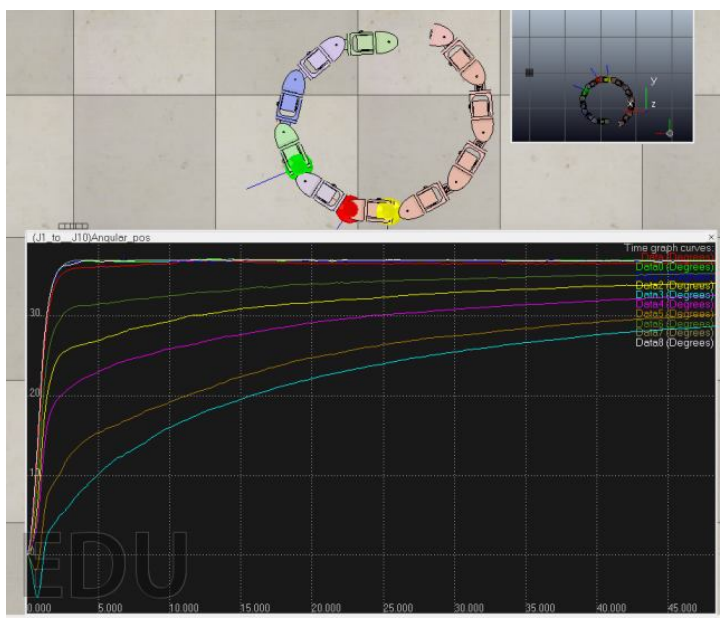

Figure 9: Circular body configuration with every joint angle of $\theta=\sum_{j=1}^{N} \theta_{j}= \pm 33.6^{n}$

\section{Circular configuration transition cyclic shape shifter using sine as oscillator}

To morph progressively from convex and concave of circular configuration, an oscillator that return the value from one to negative one (from 1 to -1 ) is needed so that a cyclic and symmetric mirroring movement can be produced. Sine wave is adopted as for producing this trajectory planning for the progressive shape changes since sine wave differential with time still produce finite value of acceleration and jerk (avoid infinite acceleration and torque). Sine wave returns cyclic value from -1 to 1 while amplitude $A$ will magnify the sine wave oscillator according to multiplication of amplitude and sine wave oscillator value. The frequency $\omega$, multiply by time $t$ decide the speed of this oscillation. Thus, the radian value produced from equation 16 will be used as amplitude which will be multiplied to sine wave oscillator with certain frequency $\omega$ and time $t$ to become equation 17 which produce a series of concave and convex movement as simulated in figure 10 and 11 which shows that this pure cyclic sine wave convex and concave movement can produce the transition of convex and concave alternate configuration where robot only stay in one place.

$$
\theta_{j}=A \sin (\omega t)=\frac{2 L}{D} \sin (\omega t)
$$

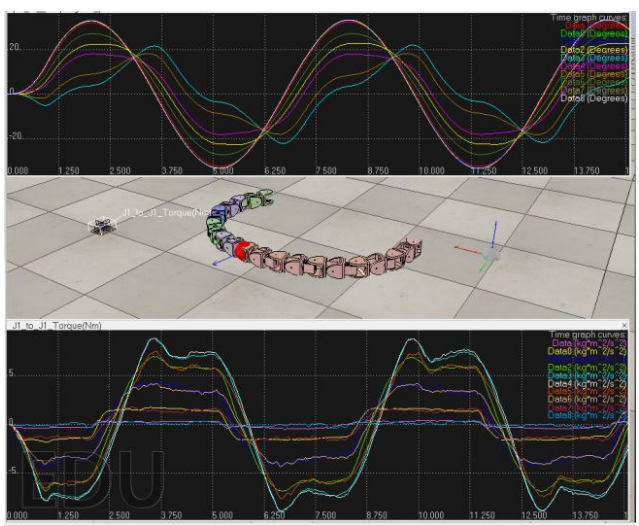

Figure 10: One (1) diameter configuration concave configuration with $D=0.6181$

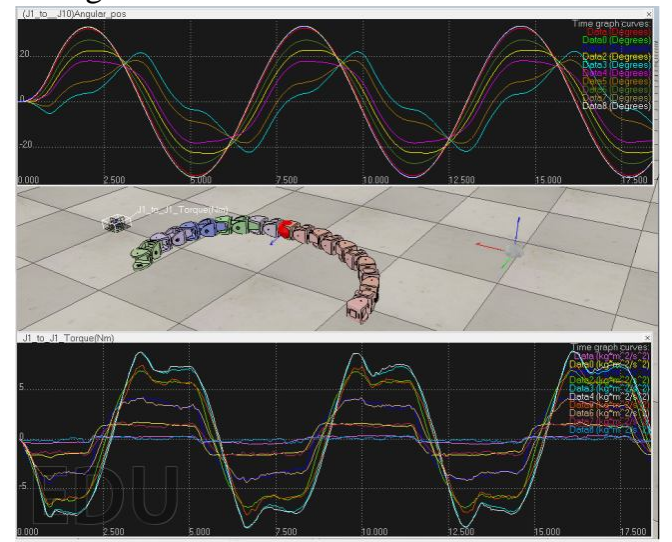

Figure 11: One (1) diameter configuration convex configuration with $D=0,6181$

\section{From circular configuration to forward Locomotion based on operational diameter}

The problem with equation 17 is no forward motion is produced beside the shape changing mirroring previous body shape configuration. In order to produce frontward motion a split circle shape or configuration need to be planned. A gradual modifier need to be added to sine wave oscillator, so the circular shape can be split gradually to opposite half configuration as depicted theoretically in figure 12. To implement gradual split circle configuration, the amplitude value produced in equation 17 also can be used again as phase angle addition to sine wave oscillator that unique to every joint which is shown in equation 18. The multiplier $j$ is related to joint number and become multiplier to amplitude value decided previously. The amplitude value when multiplied with number of local joint $\vec{j}$, actually behave as phase angle to sine wave oscillator also being called as phase angle to offset the value of angular motion in incremental manner from one joint to another. The usage of equation 17 is 
shown in equation 18 to 21 which produce individual joint angle from tail to head of snake robot belonging to joint $\mathrm{N}$.

$$
\begin{aligned}
& \theta_{i}=A \sin (\omega t+j A) \\
& \theta_{1}=A \sin (\omega t+1 ; A) \\
& \theta_{2}=A \sin (\omega t+2 * A) \\
& \theta_{N}=A \sin (\omega t+N * A)
\end{aligned}
$$

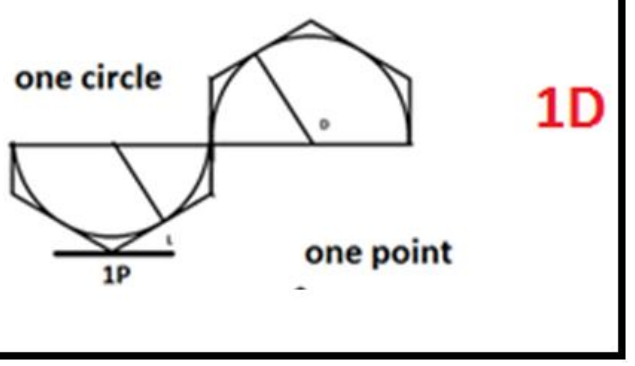

Figure 12: Theoretical one (1) diameter configuration for rectilinear gait with $D=0.6181$

\section{Translational Speed estimation}

From previous discussion, the rectilinear gait will be based on diameter $\boldsymbol{D}$ since the objective in this work is to map from wheeled locomotion or task space to gait implementation in joint space. Now we want to estimate speed based on the split circle configuration proposed in Figure 12. Thus we want to find relation from $D$ to $D_{o v}$ for speed calculation. To come up with $D_{o p}$, the total length of snake like robot will be viewed as number of virtual diameter fitting in to convex and concave cyclic progression but the circle is divided into two to produce rectilinear motion as depicted in Figure 12. One diameter configuration actually consists of convex and concave virtual circle. Equation 19 shows the concept of modelling two circles with corrected virtual diameter $D_{o p}$ to produced one diameter $\mathrm{D}$ body concave and convex configuration. The number of virtual circle desired other than one or $f$ is introduced in equation 20 which shows that for one diameter $D$ movement the value of $f$ is equal to one. Equation 21 provides the operational speed diameter $D_{o D}$ for calculating the overall speed of split circle gait.

$$
\begin{aligned}
& f\left(2 \pi D_{o p}\right)=L_{t o t} \\
& f=\frac{D}{D_{0 D}}=\frac{0.6181}{0.4}=1.545 \\
& D_{o p}=\frac{D}{2 f}=\frac{.6181}{2(1.545)}=0.2 \mathrm{~m} \\
& V_{t}=\omega \frac{D_{0 p}}{2}=\frac{L_{t o v}}{4 \pi f}=0.5 \frac{0.200}{2}=0.050 \mathrm{~m} / \mathrm{s}
\end{aligned}
$$

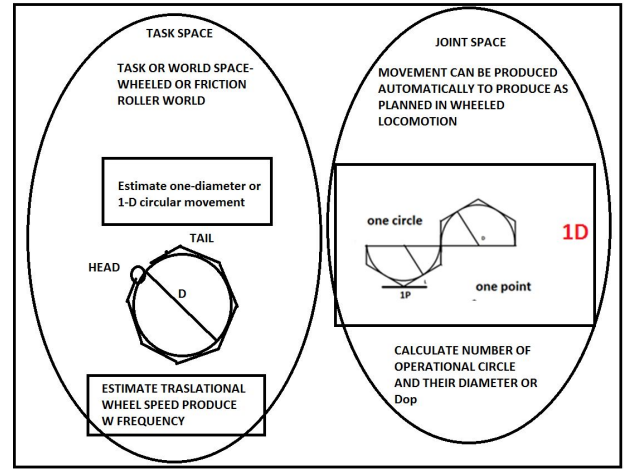

Figure 13: Mapping from wheeled locomotion or task space to gait implementation in joint space

\section{One diameter locomotion}

One wheeled diameter $D$ with angular speed $\omega=0.5 \mathrm{rad} / \mathrm{sec}$ will produce translational speed as equation 22 displayed in Figure 14 where 1-D convex configuration produce $\mathrm{X}$-movement $0.5 \mathrm{~m}$ in $28 \mathrm{sec}$ or $\mathrm{V}=0.0178 \mathrm{~m} / \mathrm{s}$ with $\mathrm{w}=0.5 \mathrm{rad} / \mathrm{sec}$ where Vtheory is 0.0773 and the maximum $\mathrm{Z}$ or height distance from ground is about $0.4 \mathrm{~m}$ which resembles theoretical operational diameter $D_{o w}$ as of 1-D diameter value of $0.30907 \mathrm{~m}$ (the differences is due to thickness of side view snake like robot profile. Progressive movement can be seen in figure 16. Equation 22 successfully produces rectilinear gait from theoretical diameter of easy to understand wheeled or friction roller locomotion with certain diameter D and angular speed $\omega$. The mapping from task space in term of wheeled locomotion to joint space in term of linkages rectilinear locomotion have been demonstrated in this simulation.

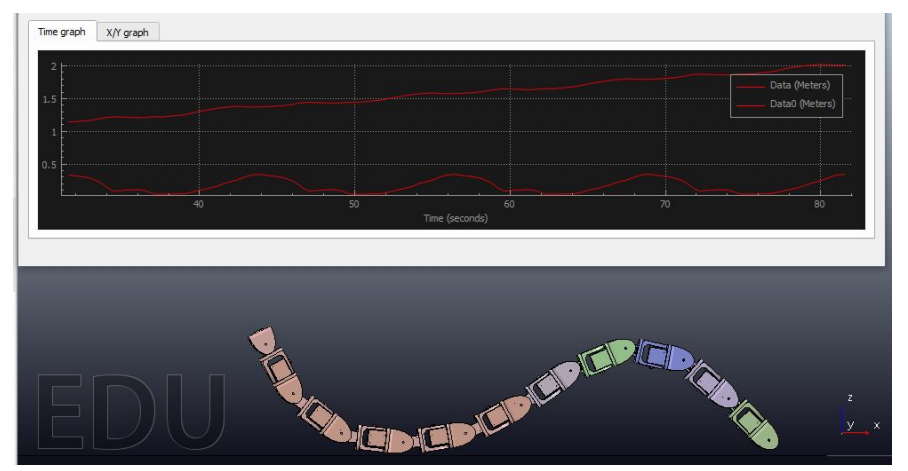

Figure 14: 1-D convex configuration produce $X$-movement $0.5 \mathrm{~m}$ in $34 \mathrm{sec}$ or $\mathrm{V}=0.0147 \mathrm{~m} / \mathrm{s}$ with $\mathrm{w}=0.5 \mathrm{rad} / \mathrm{sec}$ where Vtheory $=0.0123 \mathrm{~m} / \mathrm{s}$

\section{Operational diameter deviates from Ideal diameter}

Movement based on ideal one circle diameter suffers from low speed and high elevation joints during frontward motion since a lot of energy being used for body lifting of joints and links. The objective is to move forward but the body lifting which is not needed for forward motion need to be reduced as low as possible. Anyway, body lifting is needed to allow freedom of motion between contact points which will be discussed further in the next paragraph. The ideal diameter $D$ 
is the diameter of the whole length of snake like robot enveloping one circle. Deviation from this diameter is the ratio $f$ of ideal diameter to intended diameter $D_{i}$ as proposed in equation 26. Let say the desired diameter $D_{d}$ is half than ideal diameter which makes the total length of snake like robot in linkages world will be divided into two circle system implementing rectilinear gait doing forward motion illustrated in figure 16. This decision create the value of ratio $f$ become to 2 and produce the operational diameter $D_{o p}$ of $0.1545 \mathrm{~m}$ as in equation 28 which will yield speed approximation of about $0.0386 \mathrm{~m} / \mathrm{s}$ as in equation 29 . The actual result of half diameter locomotion is shown in Figure 16 where 0.5 -D configuration produce $\mathrm{X}$-movement $1 \mathrm{~m}$ in $30 \mathrm{sec}$ or $\mathrm{V}=0.0333 \mathrm{~m} / \mathrm{s}$ with $\omega=0.5 \mathrm{rad} / \mathrm{sec}$ where the theoretical speed is $V_{\mathrm{t}}=0.0386 \mathrm{~m} / \mathrm{s}$ (speed deviation about $16 \%$ ).The speed also affect the differences between estimation and actual speed. Figure 17 shows half-D configuration produce $\mathrm{X}$-movement $0.25 \mathrm{~m}$ in $30 \mathrm{sec}$ but with lesser frequency value of $\mathrm{w}=0.1 \mathrm{rad} / \mathrm{sec}$ where actual speed is $\mathrm{V}=0.00833 \mathrm{~m} / \mathrm{sec}$ and theoretical speed is $V_{t}=0.00773 \mathrm{~m} / \mathrm{s}($ deviation of $7.76 \%)$. The more accurate result is observed when speed is slower due to less dynamic torque dissipated to implement dynamic speed requirement. The experiment with desired diameter from 0.125 to $0.4 \mathrm{~m}$ with $\boldsymbol{\omega}=\mathbf{1} \mathrm{rad} / \mathrm{sec}$ is summarized in table 1 , while table two shows the simulation result for $\omega=0.5 \mathrm{rad} / \mathrm{sec}$.

$$
\begin{aligned}
& f=\frac{D}{D_{s i}}=2 \\
& f\left(2 \pi D_{o v}\right)=L_{t o t} \\
& D_{o p}=\frac{L_{t o t}}{2 \pi f}=\frac{1.942}{2 \pi 2}=0.15454 \mathrm{~m} \\
& V_{t}=\omega \frac{D_{o p}}{2}=\omega \frac{L_{t o t}}{4 \pi f}=0.5 \frac{1.942}{8 \pi}=0.0386 \mathrm{~m} / \mathrm{s} \\
& V_{t}=\omega \frac{D_{o p}}{2}=\omega \frac{L_{t a t}}{4 \pi f}=0.1 \frac{1.942}{8 \pi}=\mathbf{0 . 0 0 7 7 3 m} / \mathrm{s}
\end{aligned}
$$

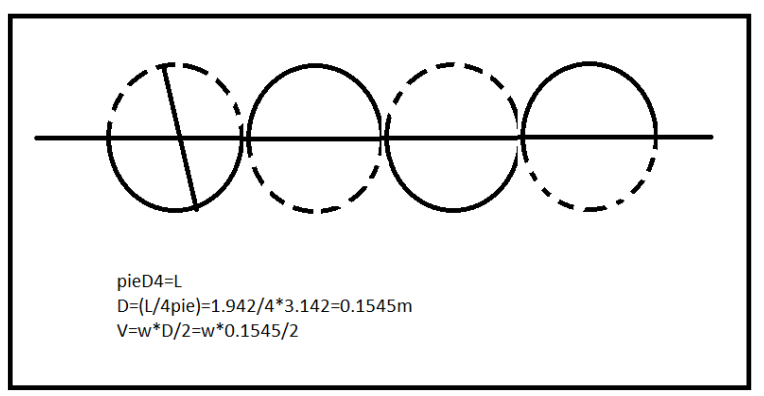

Figure 15: Friction roller operational diameter

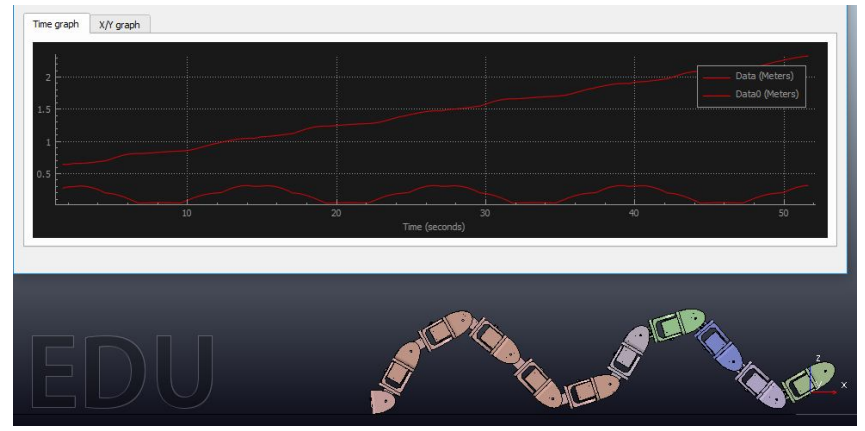

Figure 16: $0.5-\mathrm{D}$ convex configuration produce $\mathrm{X}$-movement $1 \mathrm{~m}$ in $30 \mathrm{sec}$ or $\mathrm{V}=0.0333 \mathrm{~m} / \mathrm{s}$ with $\mathrm{w}=0.5 \mathrm{rad} / \mathrm{sec}$ where

$V_{t}=0.0386 \mathrm{~m} / \mathrm{s}$

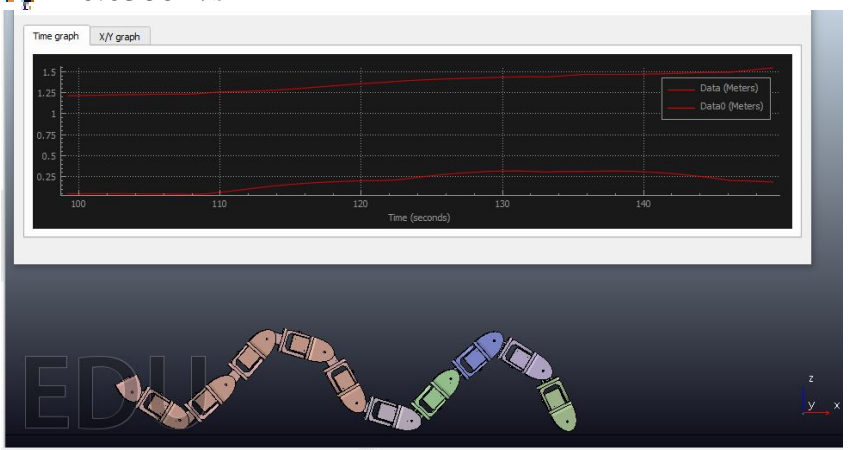

Figure 17: Half-D configuration produce $X$-movement 0.25 $\mathrm{m}$ in $30 \mathrm{sec}$ with $\mathrm{w}=0.1 \mathrm{rad} / \mathrm{sec}, \mathrm{V}=0.00833 \mathrm{~m} / \mathrm{sec}$

$V_{t}=0.00773 \mathrm{~m} / \mathrm{s}$

Table 1: Desired diameter, speed and torque result with $\omega=1$ $\mathrm{rad} / \mathrm{sec}$

\begin{tabular}{|l|c|l|l|l|l|ll|}
\hline Dd(m) & f & Doper & Vtheory(cm/s) & $\begin{array}{l}\text { Dactual } \\
(\mathrm{m})\end{array}$ & $\begin{array}{l}\text { Vactual } \\
(\mathrm{cm} / \mathrm{s})\end{array}$ & $\begin{array}{l}\text { Torque } \\
\text { Max } \\
(\mathrm{Nm})\end{array}$ & $\begin{array}{l}\text { Torque } \\
\text { Init } \\
(\mathrm{Nm})\end{array}$ \\
\hline 0.4 & 1.5455 & 0.199968 & 9.9984 & 0.35 & 6.329 & 5.1 & 5.9 \\
\hline 0.375 & 1.6485 & 0.18747 & 9.3735 & 0.35 & 6.8 & 5.1 & 4 \\
\hline 0.35 & 1.7663 & 0.174972 & 8.7486 & 0.34 & 6.25 & 4 & 3 \\
0.325 & 1.9022 & 0.162474 & 8.1237 & 0.32 & 6.61 & 2.2 & 3.1 \\
\hline 0.3 & 2.0607 & 0.18747 & 9.3735 & 0.31 & 6 & 2.52 & 2.45 \\
\hline 0.275 & 2.248 & 0.137478 & 6.8739 & 0.305 & 6.25 & 4 & 1 \\
0.25 & 2.4728 & 0.12498 & 6.249 & 0.29 & 6.25 & 2.9 & 1.1 \\
0.225 & 2.7476 & 0.18747 & 9.3735 & 0.255 & 6.25 & 4.2 & 2 \\
\hline 0.2 & 3.091 & 0.099984 & 4.9992 & 0.215 & 6 & 3.1 & 0.8 \\
\hline 0.175 & 3.5326 & 0.087486 & 4.3743 & 0.22 & 6.45 & 3.1 & 0.9 \\
0.15 & 4.1213 & 0.074988 & 3.7494 & 0.22 & 8.3 & 14 & 2.5 \\
0.125 & 4.9456 & 0.06249 & 3.1245 & 0.21 & 2.5 & 31 & 2.5 \\
\hline
\end{tabular}

\section{CONCLUSION}

Table 1 shows theoretical speed as a fraction $f$ of one diameter $\boldsymbol{D}$ wheeled locomotion in task space. Started with desired diameter $D_{d}=0.6181$ which is equal to fraction of 1 to desired diameter 0.155 which is equal to fraction of 3.987 . Designer able to plan the multi number of virtual circles by deciding number of circle $f$ which produces the value of diameter $D_{o p}$ in joint or robot space. From the table, the theoretical speed almost match with actual speed when the value of desired diameter equal to 0.25 with acceptable value of lifting effort of torque $2.9 \mathrm{Nm}$ in comparison of higher value of torque with different desired diameter. So, the 
objective in this work which is to map from wheeled locomotion or task space to gait in vertical group joints in joint space already achieved especially for operational rolling diameter about $0.25 \mathrm{~m}$. The fraction of body shape deviate from ideal diameter can also be determined such as 1D and half D body configuration as in figure 14,16 and 17 . The number of contact points will be developed further in future work.

\section{REFERENCES}

[1]. Eduardo I. Cabral Jr., Christopher Lambert M. Flores, Geromin S. Nepomuceno III, Jesus Lorenzo A. Singson and Gerald P. Arada, Kinect-based Teleoperated Pseudo-Anthropomorphic Robotic Arm International Journal of Advanced Trends in Computer Science and Engineering, Vol.8 No 5, September - October 2019, pp. $2240-2245$.

https://doi.org/10.30534/ijatcse/2019/59852019

[2]. S.V.R.K.Rao, M Kaivalya, Ch Janaki Devi, M V N Raju, Enhancing those BCI interface execution on aggravate brain-controlled portable robots, International Journal of Advanced Trends in Computer Science and Engineering, Volume 7, No.6, November - December 2018, pp 159162.

https://doi.org/10.30534/ijatcse/2018/20762018

[3]. Z. Zimmermann, I. Zeidis, C. Behn, Mechanics of Terrestrial Locomotion with a Focus on Nonpedal Motion Systems, Springer, Heidelberg;1992.

[4]. J. Steigenberger and C. Behn, Gait generation considering dynamicsfor artificial segmented worms, ROBOTICS AND AUTONOMOUS SYSTEMS., vol. 59, 2011, pp 555-562.

[5]. S.Hirose. Biologically Inspired Robots:Snake like locomotors and manipulators,Oxford University Press, Oxford 1993.

[6]. S.Ma Analysis of snake movement forms for realization of snake like robot .on proceeding IEEE international Conference Robotics and Automation,Detroit MI USA Volume 4. May 1999,pp3007-3013.

[7]. Ryo Ariizumi and Fumitoshi Matsuno,Dynamical Analysis of Sidewinding Locomotion by a snake like Robot on proceeding IEEE international Conference Robotics and Automation,Karlsruhe Germany May 2013,pp5149-5154.

https://doi.org/10.1109/ICRA.2013.6631313

[8]. Zhelong, WangQin, GaoHongyu Zhao. CPG-Inspired Locomotion Control for a Snake Robot Basing on Nonlinear Oscillators.Journal of Intelligent \& Robotic Systems.February 2017, Volume 85, Issue 2, pp 209-227.

[9]. Chaohui Gong, Ross L. Hatton and Howie Choset Conical Sidewinding 2012 IEEE International Conference on Robotics and Automation RiverCentre, Saint Paul, Minnesota, USA May 14-18, 2012.

[10]. Chaohui Gong, Matthew J. Travers, Xiaozhou Fu and Howie Choset, Extended Gait Equation for Sidewinding 2013 IEEE International Conference on Robotics and
Automation (ICRA) Karlsruhe, Germany, May 6-10, 2013.

[11]. J.K. Hopkins, B.W. Spranklin, S.K. Gupta, A Survey of Snake-Inspired Robot Designs, Bioinspiration Biomimetics., vol. 4(2), 2009, p 021001.

[12]. A.A. Transeth, K.Y. Pettersen, P. Liljeback, A Survey on Snake Robot Modeling and Locomotion, Robotica., vol. 27, 1950, pp 999-1015.

[13]. A. R. McNeill, Principles of Animal Locomotion. Princeton University Press, 2003.7540

[14]. Snake Robots Market Shares, Strategies, and Forecasts, Worldwide,2015 to 2021, WinterGreen Research INC.2015.

[15]. H. Marvi, J. Bridges, and D. L. Hu, "Snakes mimic earthworms: propulsion using rectilinear travelling waves." Journal of the Royal Society, Interface / the Royal Society, vol. 10, no. 84, p. 20130188, 2013. https://doi.org/10.1098/rsif.2013.0188

[16]. M. Tesch, K. Lipkin, I. Brown, R. Hatton, A. Peck, and a. H. C.J. Rembisz, "Parameterized and scripted gaits for modular snake robots," Advanced Robotics, vol. 23, no. 9, pp. 1131-1158, 2009.

[17]. L. I. van Griethuijsen and B. A. Trimmer, "Kinematics of horizontal and vertical caterpillar crawling," The Journal of Experimental Biology, vol. 212, pp. 1455-62, 2009

[18]. A.H. Chang, M.M. Serrano, and P.A. Vela, Shape-Centric Modeling of Traveling Wave Rectilinear Locomotion for Snake-Like Robots2016 IEEE 55th Conference on Decision and Control (CDC) ARIA Resort \& Casino December 12-14, 2016, Las Vegas, USA.

[19]. Wenbin Tang, Fabian Reyes and Shugen Ma Study on Rectilinear Locomotion Based on a Snake Robot with Passive Anchor2015 IEEE/RSJ International Conference on Intelligent Robots and Systems (IROS) Congress Center Hamburg Sept 28 - Oct 2, 2015. Hamburg, Germany 67 - 3809 TA TEHNICA CORVINIENSIS Bulletin of Engineering.

[20]. Raisuddin Khan, M. Watanabe and A.A. Shafie Kinematics Model of Snake Robot Considering Snake Scale- American Journal of Applied Sciences 7 (5): 669-674, 2010 ISSN 1546-9239 () 2010Science Publications.

[21]. Md. Raisuddin Khan, Mad Helmi Ab Majid, Shahrul Na'im Sidek, "A Snake Robot with Mixed Gaits Capability," The 4th KKU International Engineering Conference 2012 (KKU-IENC 2012) .

[22]. Norzalilah Mohamad Nor and Shugen Ma, Body Shape Control of a Snake-like Robot Based on Phase Oscillator Network, Proceeding of the IEEE International Conference on Robotics and Biomimetics (ROBIO) Shenzhen, China, December 2013.

[23]. Norzalilah Mohamad Nor and Shugen Ma, CPG-based Locomotion Control of a Snake-like Robot for Obstacle Avoidance, 2014 IEEE International Conference on Robotics \& Automation (ICRA) Hong Kong Convention and Exhibition Center May 31 - June 7, 2014. Hong Kong, China. 
[24]. Zoran Pandilov and Vladimir Dukovski Comparison Of The Characteristics Between Serial And Parallel Robots Acta Tehnica Corviniensis - Bulletin of Engineering Tome VII [2014] Fascicule 1 [January - March] ISSN: 2067 - 3809 AC e VII [2014] Fascicule 1 [January March]ISSN: $2067-3809$.

[25]. Hopkins, J. K. and Gupta, S. K., 2012, "Characterization of Forward Rectilinear-Gait Performance for a Snake-Inspired Robot," In the Proceedings of the 2012 Performance Metrics for Intelligent Systems (PerMIS'12) Workshop, College Park, MD. https://doi.org/10.1145/2393091.2393119

[26]. J.K. Hopkins, and S.K. Gupta, Design and Modeling of a New Drive System and Exaggerated ectilinear-Gait for a Snake-Inspired Robot,J. Mechanisms Robotics., vol. 6(2), 2014, pp 1138-1146.

[27]. Wenbin Tang and Shugen Ma Analysis of Rectilinear Motion of a Three-Segment Snake Robot Under Action of Dry Friction 2015 IEEE International Conference on Robotics and Automation (ICRA) Washington State Convention Center Seattle, Washington, May 26-30, 2015.

[28]. Juan Gonzalez-Gomez, Houxiang Zhang, Eduardo Boemo1 and Jianwei Zhang,Locomotion Capabilities of a Modular Robot with Eight Pitch-Yaw-Connecting Modules.www.iearobotics.com.

[29]. Wright, C., Buchan, A., Brown, B., Geist, J., Schwerin, M., Rollinson, D., Tesch, M. and Choset, H., 2012, "Design and Architecture of the Unified Modular Snake Robot," In the 15th Proceedings of the 2012 IEEE International Conference on Robotics and Automation (ICRA), Saint Paul, MN.

[30]. Li Chena, Shugen Mab, Yuechao Wangb, Bin Lib, Dengping Duana .Design and modelling of a snake robot in traveling wave locomotion.Mechanism and Machine Theory.Volume 42, Issue 12, December 2007, Pages 1632-1642.

[31]. Motoyasu Tanaka and Kazuo Tanaka Control of a Snake Robot for Ascending and Descending Steps, IEEE TRANSACTIONS ON ROBOTICS, VOL. 31, NO. 2, APRIL 2015

[32]. Florian Enner, David Rollinson and Howie Choset Simplified Motion Modeling for Snake Robots Robotics and Automation (ICRA), 2012 IEEE International Conference on, 4216-4221.

https://doi.org/10.1109/ICRA.2012.6225163 